\title{
BILAN DU PROGRAMME « POISSONS MIGRATEURS » DU CONTRAT DE PLAN ÉTAT-RÉGION 1994-1999 EN BRETAGNE
}

\author{
M.A. ARAGo (1), V. VAUCLIN (2)
}

(1) Ouest Grands Migrateurs, 149, rue d'Antrain, $35700^{\circ}$ Rennes.

(2) Conseil Supérieur de la Pêche, Délégation Régionale Champagne-Ardennes, Lorraine, Alsace - 18, rue de Nomeny, 57950 Montigny-les-Metz (anciennement chargé de mission à Ouest Grands Migrateurs de 1994 à 1999).

\section{RÉSUMÉ}

Cet article présente les réalisations du programme " poissons migrateurs " du contrat de plan Etat-Région Bretagne sur la période 1994-1999. Le programme porte sur quinze bassins versants bretons dont cinq concernés par l'espèce anguille (Anguilla anguilla), huit par le saumon atlantique (Salmo salar) et deux cours d'eau à objectif plurispécifique. Les réalisations sont présentées selon une répartition en six thèmes principaux, auxquels se rajoute le noyau central d'animation et de coordination. Les actions menées sont précisées avec une rapide comparaison des réalisations et du programme initial, qui conclut à un écart modéré en partie imputable à l'imprécision du programme initial et à une progression des connaissances sur l'état et l'écologie des deux principales espèces migratrices. Les grands axes du programme suivant (2000-2006) sont évoqués.

Mots-clés : Bretagne, contrat de plan Etat-Région, saumon atlantique, anguille, programme de restauration.

\section{ASSESSMENT OF THE PROGRAMM IN FAVOUR OF « MIGRATORY FISH »OF THE CONTRAT DE PLAN ETAT-RÉGION 1994-1999 IN BRITTANY.}

\section{ABSTRACT}

This article presents the realizations of the program in favour of the " migratory fish " of the contrat de plan Etat-Région (contract between the french state and the region of Brittany) over the period 1994-1999. The program covered fifteen river systems of Brittany, five being devoted to the european eel (Anguila anguila) and eight concerning the atlantic salmon (Salmo salar), the two remaining aiming at the restoration of more than one species of migratory fish. The operations are presented first according to the six main themes developped, the seventh being the coordination of the program itself. A short comparison of the effective realizations and the previsions is given, from which a moderate 
difference to the prevision can be concluded, due to an imprécision of the initial program and a progression of the knowledge of the two main species status and ecology. The main axes of the next program (2000-2006) are given.

Key-words : Brittany, contrat de plan Etat-region, atlantic salmon, european eel, restoration program.

\section{INTRODUCTION}

La Bretagne est considérée comme une des régions de France où les populations de poissons migrateurs sont le mieux préservées. Cette opinion repose sur le fait qu'elle dispose d'environ 25 cours d'eau accueillant des populations autonomes de saumon atlantique, et que les peuplements en anguilles observés présentent encore un état satisfaisant, compte tenu du nombre relativement faible d'obstacles rédhibitoires sur les rivières. Ces dernières sont de courte longueur et en général exemptes d'utilisations industrielles, si l'on excepte les piscicultures commerciales. En revanche, la pression de l'agriculture intensive et dans certains cas de l'agro-industrie n'est pas négligeable et génère des nuisances plus diffuses et difficilement réversibles, en matière de pollution physico-chimiques (nappes, plans d'eau de retenues, rivières), d'intégrité des habitats aquatiques (simplification résultant des travaux en lit mineur) ainsi que d'altérations du régime des eaux à l'échelle des bassins versants (irrégularité, étiages plus longs et plus sévères). En dépit d'altérations perceptibles, le statut des populations des deux principaux poissons migrateurs (saumon, anguille) semble plutôt favorable. Celui des lamproies est très mal connu et la fréquentation des aloses semble limitée à un nombre réduit de rivières (Aulne, Blavet, Trieux) sans évaluation fiable du stock à ce jour.

Dans ce contexte, un programme en faveur des poissons migrateurs a été mis en œuvre sur la période 1994-1999, dans le cadre du contrat de plan Etat-Région Bretagne. Ce volet d'action est issu du «Contrat Retour aux sources » lancé par le Ministère de l'Environnement en 1992. L'ensemble de ce programme a été coordonné par un chargé de mission employé par l'Union Régionale Bretagne-Maine-Normandie des Fédérations départementales pour la Pêche et la Protection du Milieu Aquatique (groupement « Ouest Grands Migrateurs Bretagne »), en liaison avec le Conseil Supérieur de la Pêche, la Direction régionale de l'environnement (DIREN), le Conseil régional de Bretagne et la Préfecture de région (Secrétariat Général pour les Affaires Régionales). Un bilan simplifié des opérations mises en cuvre est présenté ci-dessous. II est issu d'un document contenant en outre la programmation pour la période 2000-2006 (ARAGO, 2000), où figure aussi la liste des études et rapports effectués dans le cadre du contrat de plan 1994-1999.

\section{LE CONTENU DU VOLET « POISSONS MIGRATEURS » (1994-1999)}

\section{Seize rivières}

Le programme proposé dans le cadre du volet «poissons migrateurs » du contrat de plan Etat-Région 1994-1999 intégrait les actions publiées en 1992 par le Ministère de l'Environnement et le Conseil Supérieur de la Pêche dans le programme « retour aux sources "(PORCHER, 1992), ainsi que des actions décidées ou engagées au niveau local. Les projets ont été choisis sur des bases techniques privilégiant les actions susceptibles de déboucher sur des résultats tangibles dans un délai raisonnable. Les seize rivières concernées par les actions du programme "poissons migrateurs " sont représentées sur la Figure 1. 


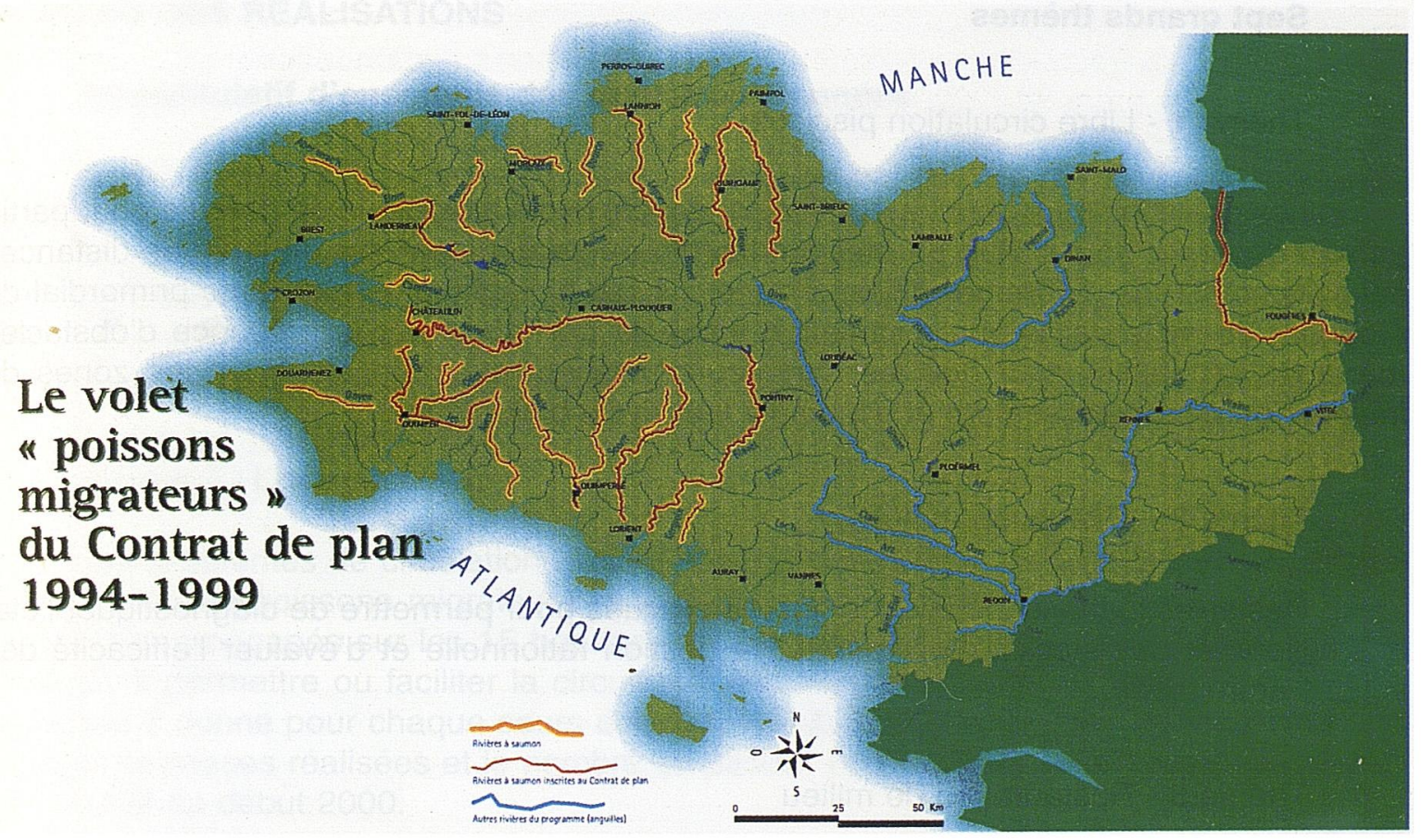

Figure 1

Les cours d'eau de Bretagne inscrits au « contrat de plan " état-région.

\section{Figure 1}

Rivers in Brittany inclued in the " contrat de plan " between the french state and the region.

\section{Deux espèces}

Le saumon atlantique constitue une richesse écologique et halieutique spécifique de la Bretagne. Avec la disparition du saumon de la majorité des grands fleuves français entre 1850 et 1950 (Rhin, Somme, Seine, Vienne et Creuse, Dordogne et Garonne) et sa raréfaction sur ses derniers bastions (Loire-Allier, Gave d'Oloron), la Bretagne est la région française où les populations de saumon atlantique sauvage sont le mieux préservées. Elle possède la plus grande densité de cours d'eau à saumons (25 rivières à saumons sur les 4 départements). Depuis l'instauration de la déclaration obligatoire des captures en 1987, on constate que la Bretagne fournit de 50 à $80 \%$ des prises annuelles de saumon atlantique en France. Cette situation assez favorable induit une responsabilité patrimoniale et requiert une véritable vigilance dans la gestion des bassins versants.

Languille européenne compte parmi les espèces principales des cours d'eau bretons où elle peut constituer plus de $50 \%$ de la biomasse sur leurs parties inférieures. Mais pour certains cours d'eau, l'effet des barrages est très sensible, car on y constate une chute importante des densités en amont des grands barrages. L'espèce est en forte régression sur l'ensemble de son aire de répartition européenne et pose aux scientifiques et aux gestionnaires des questions à ce jour non résolues. A l'heure où l'anguille se raréfie partout en Europe, et où elle subit un ensemble de facteurs adverses (obstacles à la migration, parasites, surpêche, pollution), il a paru important de favoriser au mieux la colonisation de toutes les surfaces en eau pour accroître la production de géniteurs. L'objectif est de limiter ainsi les risques d'effondrement du stock, qui poserait un véritable problème économique (pêche professionnelle) et écologique. 


\section{Sept grands thèmes}

\section{Thème 1 - Libre circulation piscicole}

Les poissons migrateurs amphihalins, dont le cycle biologique se déroule pour partie en eau douce et pour partie en mer ont besoin de se déplacer sur de longues distances pour accomplir les différentes phases de leur cycle biologique. II était donc primordial de proposer de restaurer la libre circulation piscicole perturbée par la présence d'obstacles (barrages ou seuils) pour que les migrateurs puissent atteindre aisément les zones de frayères ou de grossissement et accomplir leur cycle biologique.

\section{Thème 2 - Évaluation et suivi}

Des investigations techniques ont été prévues pour permettre de diagnostiquer l'état des milieux et des stocks, d'en orienter la gestion rationnelle et d'évaluer l'efficacité des actions entreprises.

\section{Thème 3 - Restauration de milieu}

Dans le cadre du contrat de plan 1994-1999, cette action a été limitée à quelques expériences de diversification d'habitat sur certains secteurs de cours d'eau. Des opérations de restauration-entretien se font dans le cadre d'autres partenariats dans les quatre départements bretons.

\section{Thème 4 - Soutien des effectifs en saumon}

Ces interventions, considérées comme temporaires, trouvent leur place sur des rivières en difficulté ou en cours de restauration. Elles ont été prévues pour décroître puis disparaître dès que les populations concernées retrouvent un niveau jugé suffisant.

\section{Thème 5 - Communication}

Diverses opérations telles que la réalisation de vidéos, de plaquettes ou de colloques ont été intégrées au programme, afin de faire connaitre les actions entreprises et sensibiliser le public à la thématique des poissons migrateurs et des cours d'eau.

Thème 6 - Valorisation et gestion de la ressource

Au niveau du contrat de plan, un certain nombre d'études ont été menées afin de conforter les programmes de restauration en cours et fournir au Comité de Gestion des Poissons Migrateurs des éléments nécessaires à la gestion des stocks.

\section{Thème 7 - Animation et coordination}

Lanimation et la coordination de ce programme ont été confiées à Ouest Grands Migrateurs Bretagne groupement de l'Union régionale des fédérations de pêche et de protection du milieu aquatiques de Bretagne, Maine, Normandie. L'objectif était d'assurer le lien entre les différents organismes financeurs, les maîtres d'ouvrage, les administrations et les organismes scientifiques. 


\section{LE BILAN DES RÉALISATIONS}

\section{Récapitulatif d'ensemble des opérations menées}

Cent soixante cinq actions (dossiers administratifs) ont été réalisées ou engagées, portées par 22 maîtres d'ouvrages : collectivités piscicoles (Fédérations Départementales de pêche et de protection du milieu aquatique), collectivités territoriales (Conseil Général des Côtes d'Armor, Syndicat Mixte d'Aménagement Touristique de l'Aulne et de l'Hyères, l'Institution d'Aménagement de la Vilaine, l'Institution du canal d'llle-et-Rance MancheOcéan Nord et le Pays d'accueil du Blavet).

\section{Thème 1 : Libre circulation}

Les problèmes de circulation avaient été pointés comme un des principaux facteurs limitant pour les poissons migrateurs. Il était prévu que la totalité des barrages ou seuils non encore aménagés sur les 15 bassins versants inscrits au programme soient équipés de façon à permettre ou faciliter la circulation des migrateurs, soit au total 113 ouvrages. La Figure 2 donne pour chaque cours d'eau, le nombre de passes prévues initialement, le nombre de passes réalisées et le nombre de passes dont le financement est engagé mais non réalisé au début 2000.

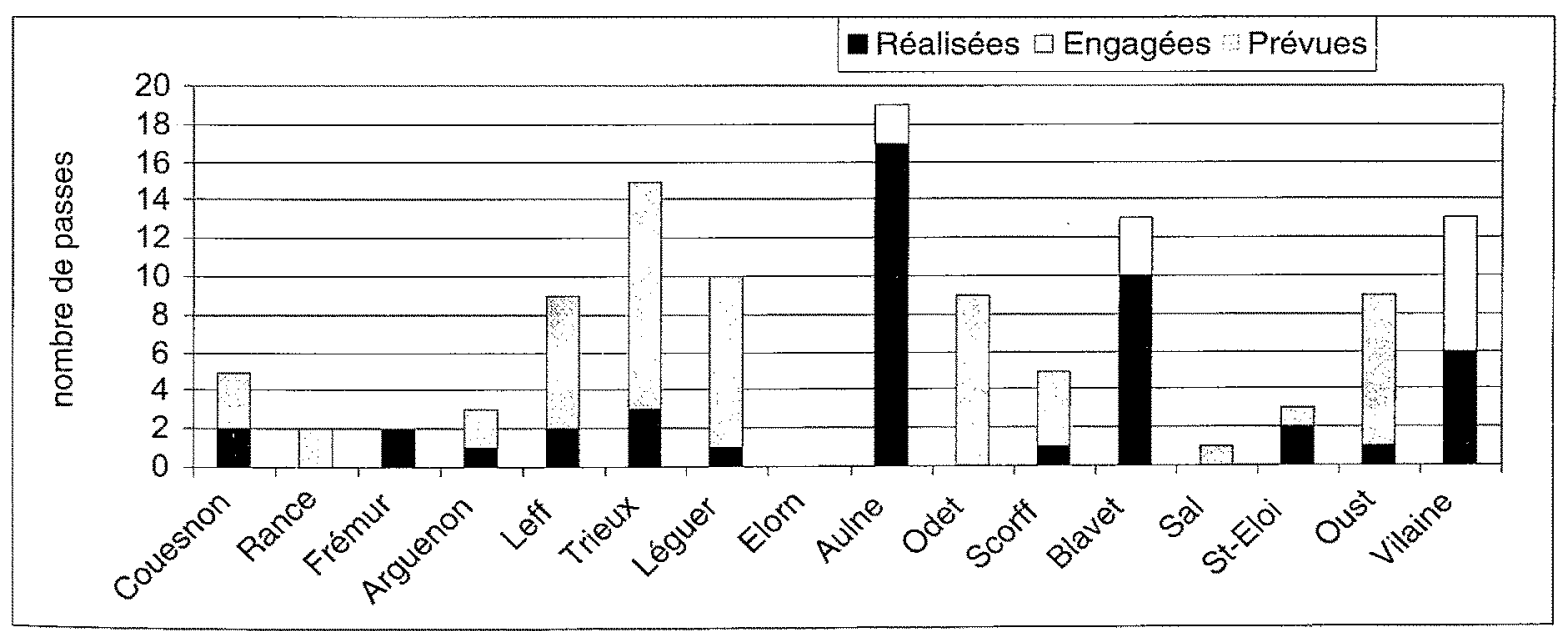

Figure 2

Nombre de passes à poissons prévues sur les seize cours d'eau du « contrat de plan ».

\section{Figure 2}

Number of fish-pass foreseen on the sixteen river of the « contrat de plan ».

De 1994 à 1999, 48 passes à poissons ont été construites, dont 14 passes à anguilles. II n'y a eu qu'un seul cas d'effacement de barrage, celui de Kernansquillec (financement hors contrat de plan). Ce faible taux de réalisation par rapport au programme initial est dû :

- à la difficulté de trouver des partenaires intéressés par la maîtrise d'ouvrage des projets. Sur certains bassins versants, la mise en place récente de structures (communauté de commune, syndicat de bassin versant...) motivées par la restauration des milieux aquatiques et des problèmes de migration devrait, dans l'avenir, faire avancer plus rapidement ces dossiers ; 
- au fait que la nécessité biologique d'aménager certains ouvrages est relativisée au vu des bons résultats obtenus lors des pêches par indices d'abondance des juvéniles de saumon en amont des barrages ou seuils présents sur certains cours d'eau.

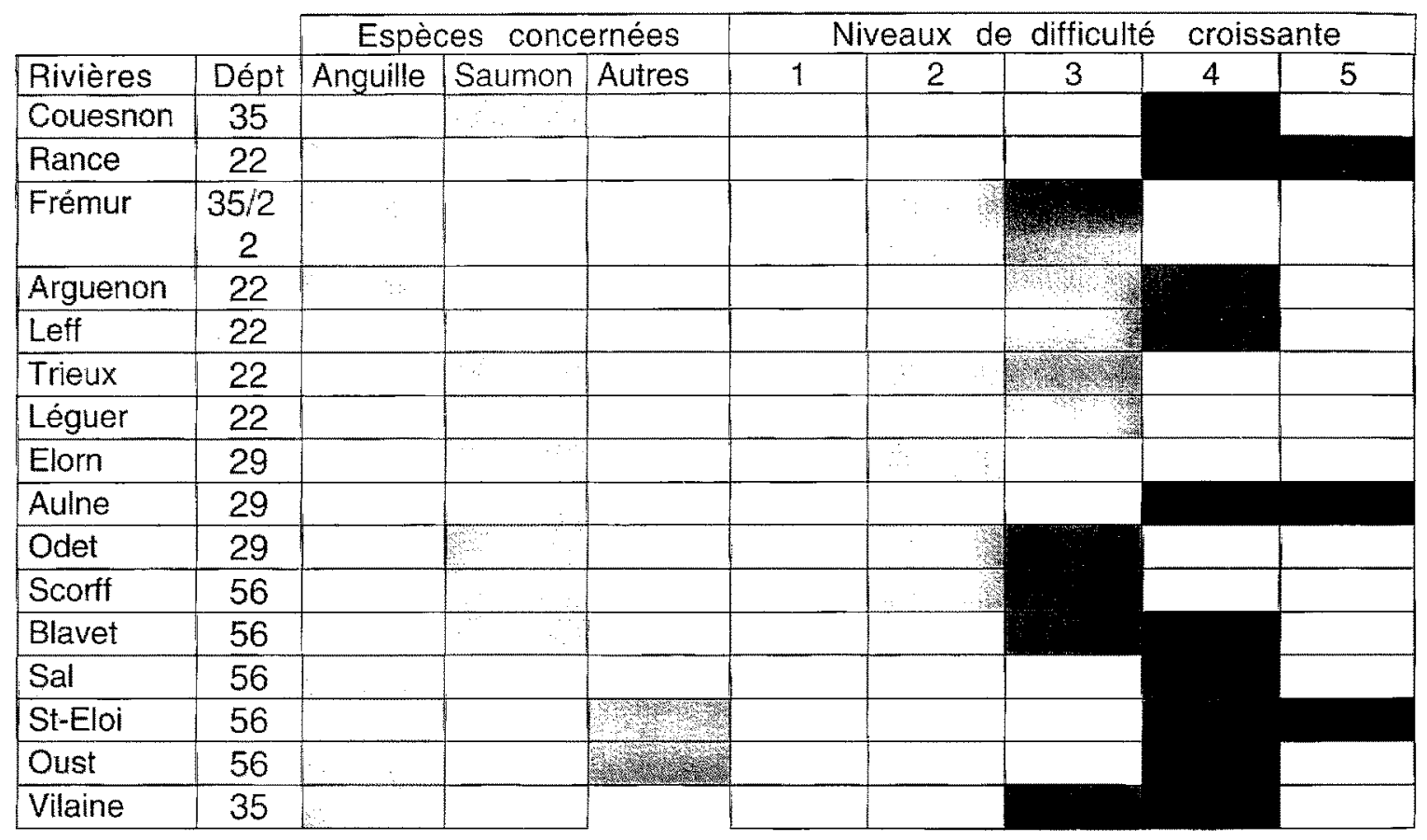

\begin{tabular}{|l|l|ll}
\hline 1 & totalement libre & 2 & fluide \\
\cline { 2 - 3 } & blocages importants & 5 & Blocage total
\end{tabular}

\section{Figure 3}

Evaluation de l'état de la circulation des poissons migrateurs par cours d'eau à la fin du programme, en 1999.

\section{Figure 3}

State of the migrating possibilities for the migratory fish per river at the end of the program, in 1999

\section{Thème 2 : Évaluation et suivi}

Ce thème assez vaste regroupe plusieurs types d'actions.

\section{Cartographie d'habitats à juvéniles de saumon}

Les zones d'habitats favorables au développement de juvéniles de saumon ont été identifiées selon des critères hydrauliques, de vitesse et de profondeur d'eau. Les zones de production de juvéniles sont essentiellement les zones de radier, de rapide voire de plats courants, peu profonds à fond grossier. La cartographie de ces zones permet de quantifier le potentiel d'habitat présent sur les cours d'eau. Cette donnée peut ensuite être traduite en nombre de smolts potentiel, lui-même théoriquement convertissable en un nombre de saumons adultes de remontée. Cette cartographie permet en outre:

- de déterminer l'intérêt de restaurer le saumon sur un bassin ou une partie de bassin, notamment pour définir les priorités d'aménagement en passes à poissons en fonction du potentiel d'habitat disponible; 
- de proposer des modalités d'exploitation liées à la capacité de production à l'aide de la méthode des TAC (Total Autorisé de Captures - PREVOST et PORCHER, 1996);

- de localiser les zones de production afin de mieux les protéger s'il en était besoin.

Les rivières et certains de leurs affluents cartographiées sont les suivantes : Couesnon, Rance, Arguenon, Leff, Trieux, Jaudy, Léguer, Douron, Elorn, Aulne, Odet, Scorff, Blavet, Sal, St-Eloi, Arz, Claie, Trévelo. Les surfaces d'habitat favorables ainsi recensées et les superficies de bassin versant sont reportées sur la Figure 4 . Ce graphique permet de repérer les cours d'eau ayant un potentiel de production de juvéniles de saumon important par rapport à la superficie du bassin versant, tels que le Trieux, le Léguer, le Douron, l'Elorn, l'Odet, le Scorff.

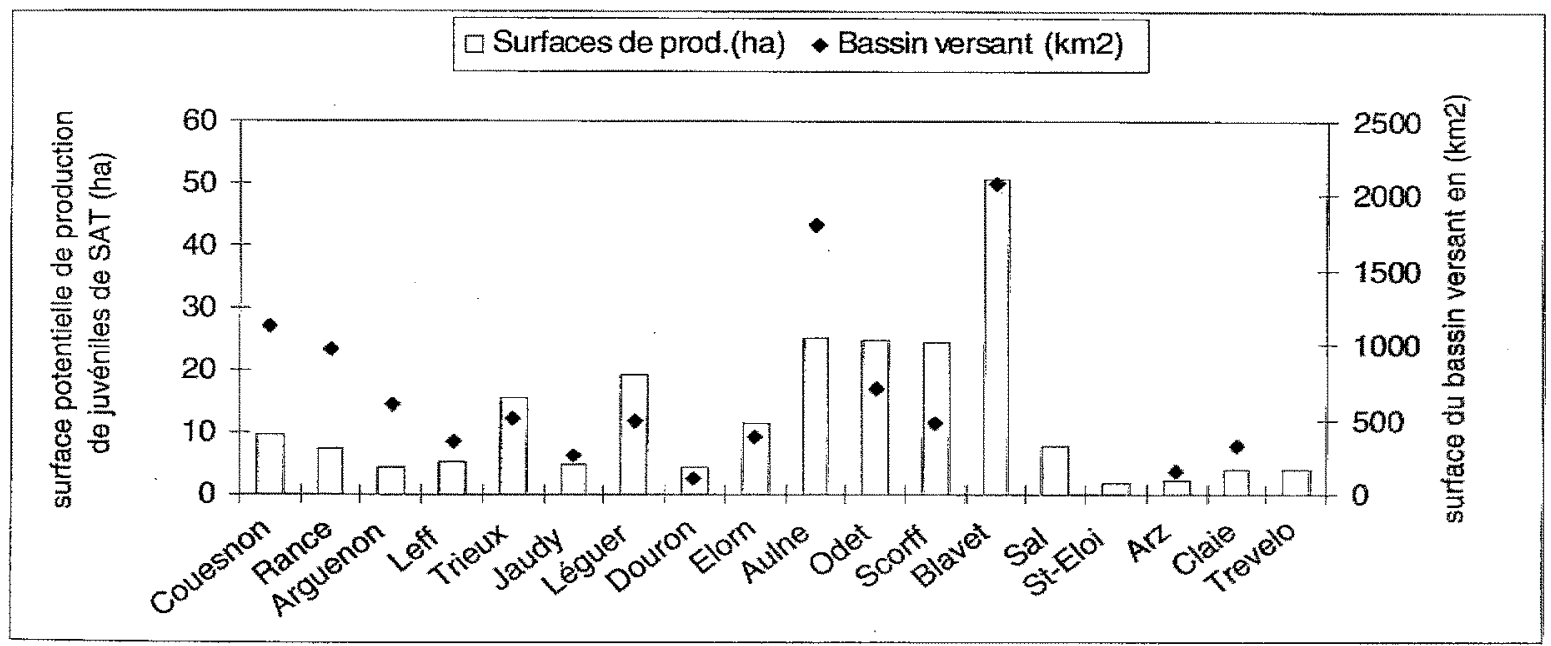

\section{Figure 4}

Surfaces de production pour les jeunes saumons par rivière.

\section{Figure 4}

\section{Surface of young salmon breeding grounds per river system.}

\section{indice d'abondance}

L'évaluation des populations automnales de juvéniles de saumon est réalisée à travers les indices d'abondances. La méthode vise à évaluer la population automnale de tacons (juvéniles de l'année) sur les différents bassins versants suivant le protocole établi par PRÉVOST et BAGLINIĖRE (1993). Le protocole de pêche, moins astreignant que les inventaires classiques par pêche électrique permet d'échantillonner un plus grand nombre de points. On obtient ainsi une évaluation plus complète de la répartition des juvéniles sur l'ensemble du bassin étudié.

Cette méthode d'échantillonnage a été généralisée à partir de 1997, à 7 cours d'eau : le Leff, le Léguer, le Trieux, l'Aulne, les rivières de Quimper, le Blavet et les affluents de l'Oust. Trois autres cours d'eau ont complété ce suivi à partir de 1998 : l'Elorn, le Douron et le Couesnon. Le Scorff est aussi échantillonné par indice d'abondance dans le cadre d'un programme de recherche global sur la dynamique de population du saumon. $C$ e vaste réseau regroupant près de 150 points d'échantillonnage a permis d'obtenir une évaluation annuelle de la reproduction naturelle et du taux d'implantation des saumons déversés. Les indices d'abondance pour les cours d'eau échantillonnés en 1997 et 1998 sont reportés sur la Figure 5 . On remarque des fluctuations inter-annuelles très importantes. 


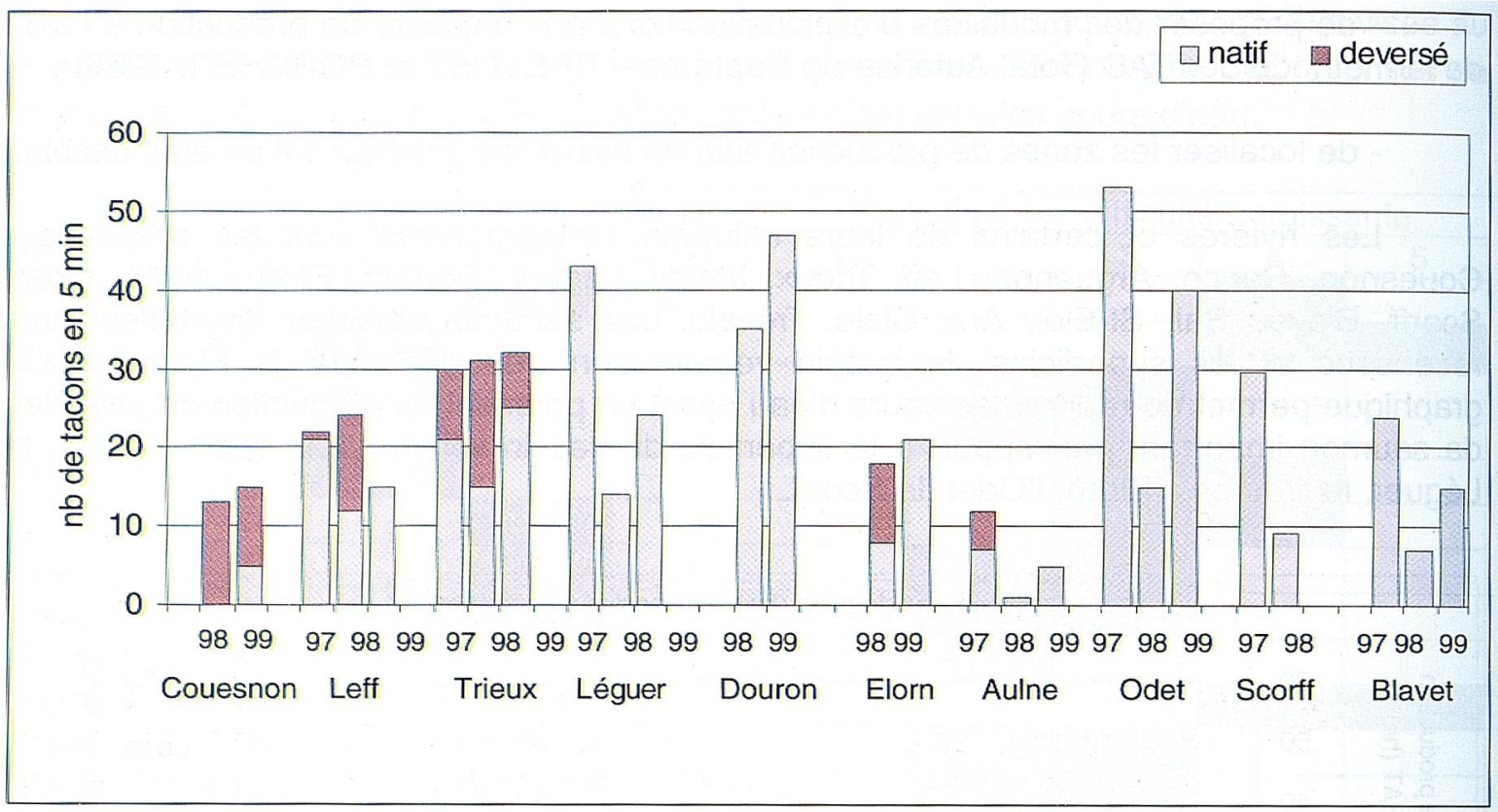

Figure 5

Indices d'abondance de saumons en 1997 et 1998.

\section{Figure 5}

\section{Young salmon indexes of abundance in 1997 and 1998.}

\section{Les suivis de migration}

Le suivi de migration mis en ceuvre au niveau d'un ouvrage de franchissement doit permettre de connaître précisément les flux entrants et sortants (quand cela est possible) pour analyser la dynamique de la population étudiée.

Le suivi de migration de saumons a été mis en cuvre sur deux cours d'eau : la Loysance (affluent du Couesnon) et le Scorff. Des piégeages en continu ont permis de dénombrer et mesurer la taille des poissons migrants. En outre le traitement de l'enregistrement vidéo réalisé au niveau de la passe à poissons du barrage d'Arzal permet de dénombrer les différentes espèces accédant au bassin de la Vilaine.

L'exemple de la Loysance, sur le bassin du Couesnon donne des résultats intéressants. Le suivi des migrations montre un taux de retour de saumon important sur la Loysance depuis 1994 ainsi qu'une reproduction naturelle (13\% des smolts piégés en 1998 sur la Loysance sont issus de reproduction naturelle). En 1999, aucun alevinage n'a été effectué sur la Loysance pour voir si la reproduction naturelle suffisait à saturer l'habitat disponible et ne pas concurrencer les tacons nés en milieu naturel. Les pêches automnales réalisées selon la méthode des indices d'abondance ont mis en évidence des densités d'alevins natifs supérieures à la moyenne régionale sur les principaux affluents du Couesnon, notamment sur les cinq stations prospectées par inventaire classique sur la Loysance.

Un suivi des migrations d'anguille a été mis en place sur 5 bassins (Rance, Arguenon, Frémur, Vilaine, Sal). Les objectifs concernant les suivis de migration des anguilles sont multiples:

- mettre en évidence l'importance d'un flux potentiel de migration pour justifier un aménagement spécifique pour la montaison des anguilles. Ce suivi permet aussi de bien 
cibler les périodes de migration ainsi que la taille des anguilles cherchant à franchir l'ouvrage et de dimensionner correctement la passe à anguilles (cas sur la Rance, l'Arguenon, le Sal, la Vilaine à Malon et l'Oust à la Potinais).

- étudier la dynamique de population de l'anguille sur un bassin versant. C'est l'objectif principal des études menées sur le Frémur et la Vilaine, qui constituent des bassins pilotes d'étude. L'objectif de cette action est de mettre au point des méthodes et des outils permettant aux gestionnaires de justifier leurs interventions sur des bases techniques, à l'image de ce qui se fait pour le saumon. II s'agit de définir et de préserver une cible estuarienne d'échappement (au niveau de là pêcherie) suffisante pour garantir une colonisation correcte de l'ensemble du bassin versant.

Les études effectuées depuis 1996 sur le Frémur ont permis d'acquérir des connaissances supplémentaires sur le cycle biologique de l'anguille et la dynamique de population de cette espèce. Ces résultats seront à préciser et à confirmer par la suite. Au niveau de la montaison, il est montré que les anguillettes au-dessus de $14 \mathrm{~cm}$ sont bloquées au niveau des barrages considérés jusqu'à présents comme franchissables par reptation. L'évaluation du stock en place a permis d'apprécier la structure démographique et la distribution spatiale de la population en fonction des conditions de recrutement et des paramètres environnementaux. On a ainsi pu quantifier la proportion d'anguilles argentées présentes sur le bassin, considérées comme les candidates à la dévalaison et susceptibles de participer à la reproduction en mer des Sargasses. Les premiers résultats indiquent que la proportion d'anguilles argentées présentes sur le bassin reste stable chaque année, et que seulement $20 \%$ des anguilles argentées dévalent effectivement. D'autre part un modèle de la cinétique de dévalaison permettant de prédire la période de migration sur le Frémur a été établi. L'exploitation de ces résultats devrait permettre de proposer des modes de gestion des barrages qui faciliteraient l'échappement des géniteurs (ACOU,1999).

Les études réalisées sur le Frémur, couplées avec les résultats obtenus sur l'anguille au niveau d'autres bassins versants et notamment sur la Vilaine, doivent permettre dans les années à venir de définir des outils de gestion des stocks d'anguilles. Lobjectif étant de produire un nombre optimal de géniteurs susceptibles de participer à la reproduction en mer des Sargasses.

\section{Études thématiques plus approfondies}

Des études à caractère plus ponctuel ont été menées, telles que le suivi par radiopistage des saumons sur l'Aulne (en cours au début 2000), l'étude de la sensibilité des saumons au parasite Gyrodactylus salaris, dont le risque d'introduction fait peser une grave menace sur les stocks français, ainsi qu'une étude relative à l'identification biologique et à la gestion des populations bretonnes d'aloses.

\section{Thème 3 : Restauration de milieu}

Seule une action de diversification d'habitat par pose de blocs a été menée sur le Couesnon. Cette expérience n'a pas été concluante car le principal facteur limitant sur ce cours d'eau est le faible débit d'étiage.

Thème 4 : Soutien des effectifs en saumon

Au départ, six rivières étaient concernées par les soutiens d'effectif en saumons : le Leff, le Léguer, le Trieux, l'Aulne, les rivières de Quimper et le Couesnon. Le soutien d'effectif était considéré comme une opération à caractère temporaire, à mener sur des cours d'eau dont le stock en saumon était jugé déficitaire pour relancer la reproduction 
naturelle du saumon. Les montants financiers prévus et engagés en matière d'alevinage pour chaque cours sont représentés sur la Figure 6 . Au vu des bons résultats d'indices d'abondance, qui mettent en évidence une reproduction naturelle importante, le soutien d'effectif a été arrêté à partir de 1996 sur le Léguer et sur le l'Odet, puis à partir de 1999 sur le Leff et le Trieux. Le Tableau I fait un bilan des alevinages effectués de 1994 à 1999 sur les 6 cours d'eau concernés par les soutiens d'effectif en saumon.

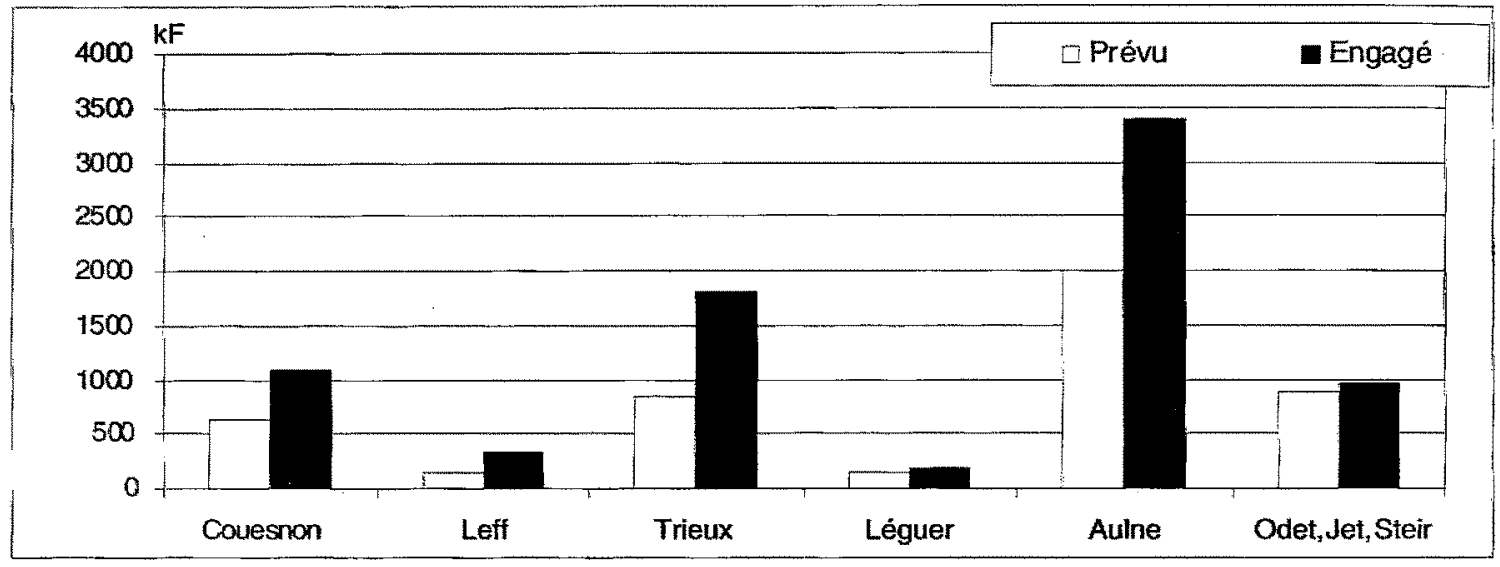

\section{Figure 6}

Montants financiers prévus et engagés pour l'alevinage en saumons de 1994 à 1999.

\section{Figure 6}

Financial amounts foreseen and spent for young salmon releases from 1994 to 1999.

Le cas de l'Aulne est particulier. L'alevinage en saumons sur l'Aulne a été très important et a dépassé les prévisions financières initiales. De 70000 à 100000 tacons et 30000 smolts ont été déversés chaque année sur ce bassin fluvial.

\section{Trois remarques :}

- le calcul du taux de retour d'adultes par rapport aux smolts déversés l'année d'avant a été fait en 1994 sur un affluent de l'Aulne, et donne le chiffre de $3,4 \%$ (NIHOUARN, comm. pers.). C'est une valeur élevée sur un strict plan technique ;

- le marquage non systématique des juvéniles avant déversement (qui s'explique en partie par les effectifs importants) et le manque de structure permettant un recueil fiable de l'information, ne permettent pas de cerner précisément la part des repeuplements dans les retours d'adultes;

- malgré le niveau élevé des déversements, on n'a pas obtenu le résultat souhaité, à savoir un recrutement significatif de juvéniles issus de la reproduction naturelle. D'autres facteurs limitants interviennent. Le plus probable est un blocage des remontées d'adultes sur la partie aval canalisée, liée à la succession de barrages et à la période de migration des castillons (eaux basses et chaudes), plus d'autres problèmes de qualité d'eau. Un effet visible de ces déversements est l'augmentation des captures à la ligne. Elles étaient inférieures à 150 prises annuelles jusqu'à 1991 et varient de 250 à 1040 prises à partir de 1992. 
Bull. Fr. Pêche Piscic. (2000) 357/358 : 357-372 - $367-$

\section{Tableau I}

Bilan des alevinages en saumon effectués de 1994 à 1999.

\section{Table I}

Total young salmon releases from 1994 to 1999.

\begin{tabular}{|c|c|c|c|c|c|c|c|}
\hline & Stade & TRIEUX & LEGUER & LEFF & AULNE & ODET & COUESNON \\
\hline \multirow[t]{4}{*}{1994} & Parrs $<6$ mois & 2500 & 21600 & ; & & & 4700 \\
\hline & Parrs $>6$ mois & 12000 & 10000 & 11300 & 100000 & 30000 & 9000 \\
\hline & $\begin{array}{l}\text { Pré-smolt et } \\
\text { smolt }\end{array}$ & 12665 & & & 30700 & & 2700 \\
\hline & & & & & & & \\
\hline \multirow[t]{4}{*}{1995} & Parrs<6mois & 15350 & 22000 & & 35000 & & 39800 \\
\hline & Parrs $>6$ mois & 62800 & 32000 & 15000 & 67000 & 32000 & \\
\hline & $\begin{array}{l}\text { Pré-smolt et } \\
\text { smolt }\end{array}$ & 22086 & 12335 & & 32710 & & 10467 \\
\hline & & & & & & & \\
\hline \multirow[t]{4}{*}{1996} & Parrs $<6$ mois & & & 16000 & & & 32730 \\
\hline & Parrs $>6$ mois & 60955 & & & 114600 & 30000 & \\
\hline & $\begin{array}{l}\text { Pré-smolt et } \\
\text { smolt }\end{array}$ & 17450 & & 3200 & 33000 & & 10467 \\
\hline & & 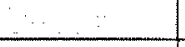 & & & & & \\
\hline \multirow{3}{*}{1997} & Parrs $<6$ mois & 23193 & & & 177972 & & 73750 \\
\hline & Parrs $>6$ mois & 35300 & & 10000 & 141645 & & \\
\hline & $\begin{array}{l}\text { Pré-smolt et } \\
\text { smolt }\end{array}$ & 11374 & & & 23019 & & 15270 \\
\hline & & & & & & & \\
\hline \multirow[t]{3}{*}{1998} & Parrs $<6$ mois & 17000 & & & & & 59650 \\
\hline & Parrs $>6$ mois & 36675 & & 15000 & 139040 & & \\
\hline & $\begin{array}{l}\text { Pré-smoit et } \\
\text { smolt }\end{array}$ & 20300 & & & 30440 & & 1640 \\
\hline \multirow[t]{4}{*}{1999} & Parrs <6mois & 8180 & & & & & 48200 \\
\hline & Parrs $>6$ mois & & & & 79500 & & \\
\hline & $\begin{array}{l}\text { Pré-smolt et } \\
\text { smolt }\end{array}$ & 21351 & & & 26500 & & \\
\hline & & & & & & & \\
\hline
\end{tabular}

Ainsi que l'ont montré PRÉVOST et PORCHER (1999) dans une analyse de l'évolution du stock de saumon sur le bassin de l'Aulne, le programme de repeuplememt réalisé a eu des effets importants:

- sur le retour d'adultes multiplié par 4 à 5 par rapport a 1980-1990:

- sur les captures réalisées multiplié par 3,75 par rapport a 1980-1990;

- sur le nombre de géniteurs potentiels restant aprés la saison de pêche.

En revanche:

- il n'a eu aucun impact sur la reconstifution d'un stock savvage, actuellement au plus bas ;

- il a généré un niveau de capture qui ne pourra pas etre foumi par un stock sauvage autonome. 
Un bilan de l'ensemble des données disponibles sur l'Aulne devrait permettre de définir une stratégie durable pour le maintien du saumon sur ce cours d'eau. La nécessité de continuer les soutiens d'effectif sur l'Aulne et le Couesnon sera examinée en fonction des résultats d'évaluations en cours sur ces rivières.

\section{Thème 5 : Communication}

Les actions menées dans ce domaine sont les suivantes : réalisation de films vidéo relatifs à la destruction du barrage de Kernansquillec (1996) et à l'équipement du barrage d'Arzal sur la Vilaine, organisation d'un colloque sur le saumon en Bretagne (1996), exposition sur l'anguille sur l'Arguenon (1998), plaquette sur le bilan des actions en faveur des poissons migrateurs en Bretagne (1999).

\section{Thème 6 : Valorisation et gestion de la ressource}

Les actions dans ce domaine d'intervention sont des enquêtes halieutiques menées sur les pêcheries récréatives du saumon (Blavet, Aulne) et sur les pêcheries civellières en estuaire (Aulne, Vilaine).

Les enquêtes halieutiques sur les pêcheurs de saumon, menées sur le Blavet et sur l'Aulne, rivières canalisées de Bretagne montrent une accumulation de l'effort de pêche sur les barrages situés en aval, qui s'explique par la meilleure capturabilité des saumons à leur entrée en eau douce et à un blocage ou un ralentissement des migrations de saumons liées aux obstacles. Un volet de valorisation et de suivi de la pêche du saumon à la ligne a mis en évidence les dépenses liées à la pêche du saumon en Finistère (et particulièrement sur l'Aulne) et leur structure. La dépense annuelle par pêcheur se situe autour de 10700 francs, dont 40 à $50 \%$ de frais de transport (BRULARD, 1995 ; BRULARD, 1998).

\section{Thème 7 : Coordination}

Ce thème s'est imposé de lui-même dès 1995, afin de mener à bien l'ensemble des actions prévues dans le contrat de plan Etat-Région de manière homogène et fidèle à l'esprit du programme. Ce choix initial s'est trouvé conforté a posteriori par l'expérience acquise progressivement en cours de programme.

\section{Bilan financier}

L'engagement global de crédits en septembre 1999 s'établit à 37,2 millions de francs, soit sensiblement plus que l'enveloppe de 32 millions de francs prévue au départ. Le Tableau II et la Figure 7 récapitulent les sommes engagées par chaque organisme dans chacun des départements bretons.

Différents partenaires ont contribué au financement du programme : le Ministère de l'environnement, Conseil Régional de Bretagne, l'Union europẻenne fonds structurels européens FEOGA et FEDER), l'Agence de l'Eau Loire-Bretagne, les Conseils Généraux des quatre départements et les différents maîtres d'ouvrage, contribuant classiquement sur une action donnée à un minimum d'autofinancement de $20 \%$.

La Figure 8 donne la répartition des sommes engagées par thème d'action. On constate que deux thèmes, l'evaluation / suivi et la libre circulation representent plus de $60 \%$ des montants engagés. Le soutien des effectifs en saumons vient en troisième position avec $23 \%$ du montant global. 
Tableau II

Montants financiers engagés par chaque partenaire.

Table II

Financial amont spent by each partner.

\begin{tabular}{|l|r|r|r|r|r|r|r|}
\hline \multicolumn{1}{|c|}{ Financeurs } & Bretagne & $\%$ & dpt 22 & dpt 29 & dpt 35 & dpt 56 & $\begin{array}{c}\text { Actions } \\
\text { régionales }\end{array}$ \\
\hline Europe & 901997 & 25 & 1072349 & 2930956 & 1000142 & 3433740 & 400900 \\
\hline Etat & $\mathbf{6 0 0 5 9 7}$ & 15 & 860380 & 1563944 & 1469517 & 1329438 & 711659 \\
\hline Région & 275854 & 8 & 120359 & 603500 & 787563 & 839888 & 324772 \\
\hline $\begin{array}{l}\text { Agence de } \\
\text { l'eau }\end{array}$ & $\mathbf{5 4 4 6 4 9 1}$ & 15 & 865700 & 1333000 & 1124400 & 1963391 & 160000 \\
\hline $\begin{array}{l}\text { Conseils } \\
\text { Généraux } \\
\text { 22,29,35,56 }\end{array}$ & $\mathbf{2 7 0 7 5 0 6}$ & 8 & 917170 & 910400 & 116576 & 763360 & \\
\hline $\begin{array}{l}\text { Collectivités } \\
\text { piscicoles } \\
\text { (FDAPPMA, cSP) }\end{array}$ & $\mathbf{8 1 0 4 3 0 6}$ & 22 & 1177608 & 4201866 & 965545 & 1299287 & 460000 \\
\hline Autres & $\mathbf{3 1 4 2 3 8 7}$ & 7 & 198400 & 157017 & 352278 & 1727552 & 592570 \\
\hline totalen francs & $\mathbf{3 7 1 8 0 ~ 1 3 8}$ & $\mathbf{1 0 0}$ & $\mathbf{5 2 1 1 9 6 6}$ & $\mathbf{1 1 7 0 0 6 9 3}$ & $\mathbf{5 8 1 6 0 2 1}$ & $\mathbf{1 1 3 5 6 6 5 6}$ & $\mathbf{3 0 9 4} 802$ \\
\hline
\end{tabular}

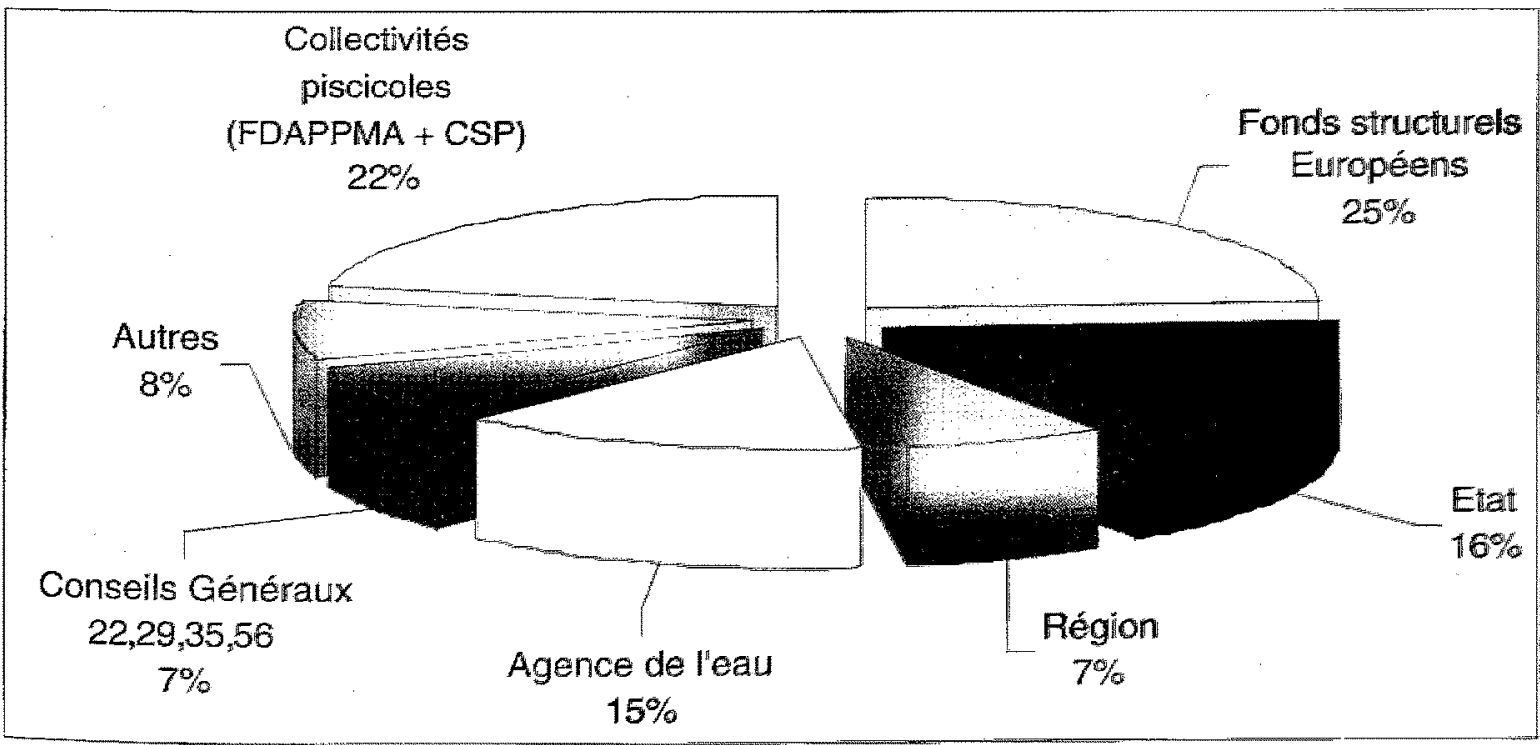

Figure 7

Participation financière des différents partenaires au volet poissons migrateurs $n$.

\section{Figure 7}

Financial contribution of the partners to the program in favour of the sigratory fish ". 


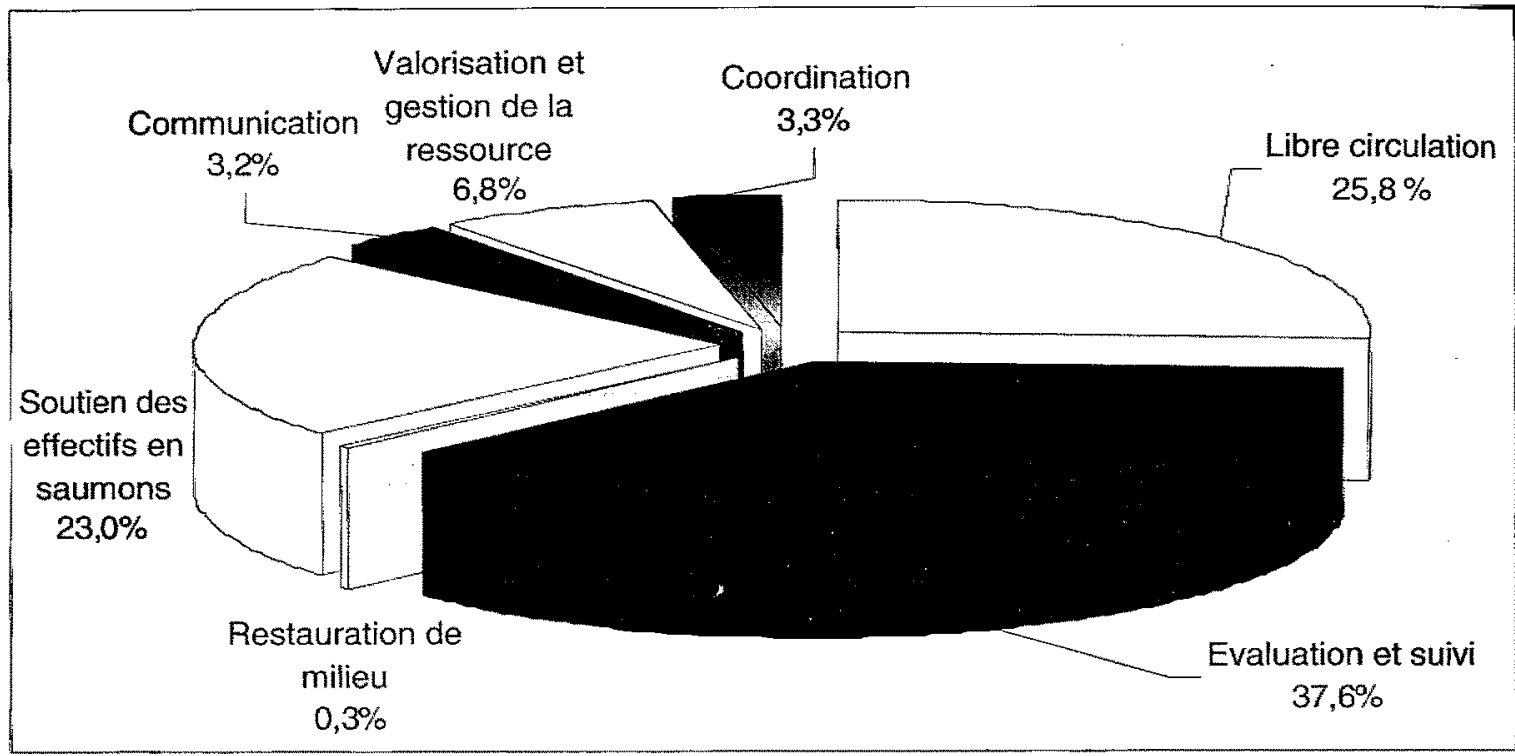

\section{Figure 8}

Répartition des crédits selon les thèmes d'actions (total : 37,2 MF).

\section{Figure 8}

Distribution of the financial supplies according to the theme of action

(total : 37,2 millions french francs).

La Figure 9 illustre la répartition des sommes engagées par cours d'eau. Les montants engagés sur la majorité d'entre eux sont inférieurs à 2 millions de francs. Sur cinq cours d'eau, le coût global des opérations menées est compris entre 2 et 4 millions de francs, sur deux autres, le coût global est supérieur à 4 millions de francs. L'Aulne, avec près de 10 millions de francs à elle seule, reflète les difficultés que pose la restauration d'une population de saumons affectée par l'accumulation des problèmes de libre circulation et de qualité d'eau auquel s'ajoutent les besoins de soutien d'effectifs et d'évaluation des actions entreprises.

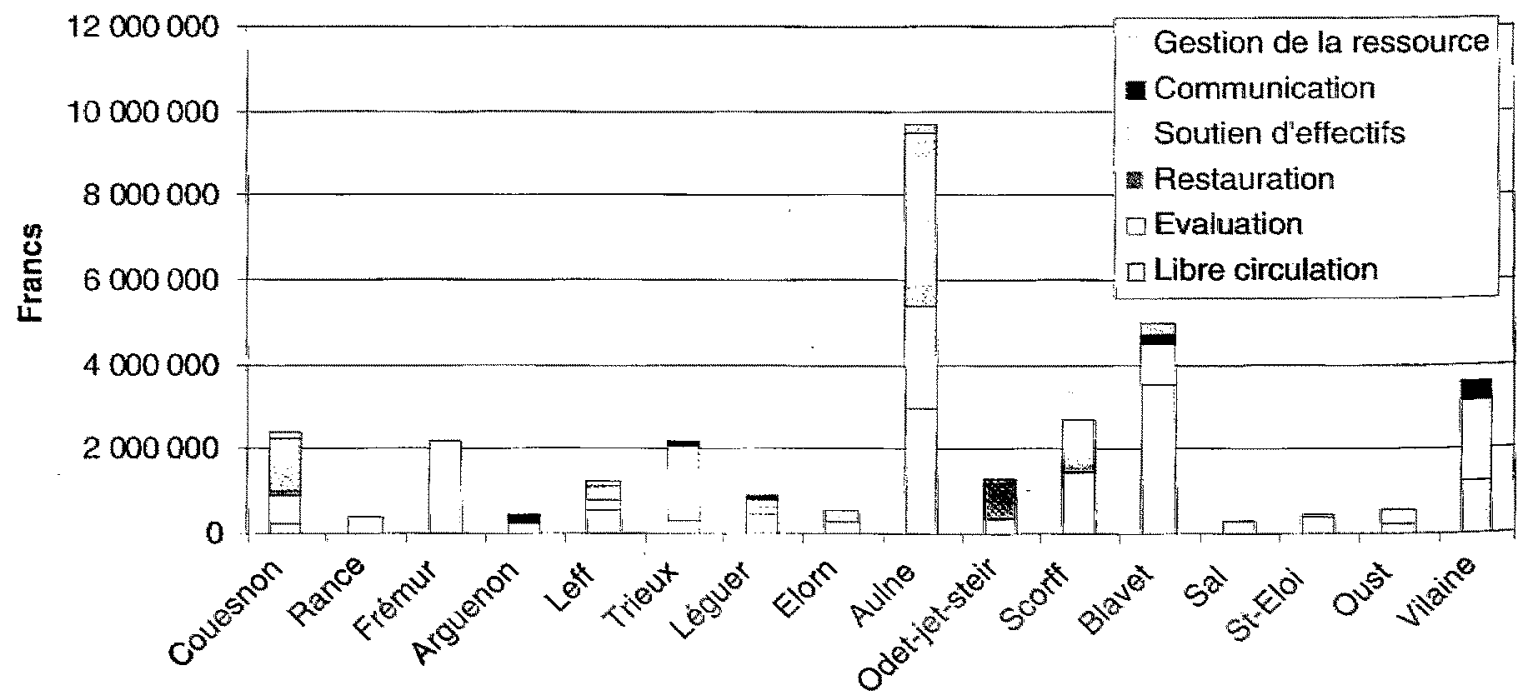

\section{Figure 9}

Montants financiers engagés par cours d'eau.

\section{Figure 9}

Financial amounts spent by river system. 


\section{CONCLUSIONS}

Le programme initial «poissons migrateurs " établi pour le contrat de plan EtatRégion Bretagne présentait une structure globalement cohérente. II était centré sur deux espèces principales : le saumon atlantique et l'anguille. Rétrospectivement, on peut considérer qu'il manquait de doctrine générale, même si ses grandes lignes étaient perceptibles à la lecture du document, le programme se limitait surtout à une liste d'actions à réaliser. II manquait aussi d'objectifs quantitatifs et chiffrés, en partie imputable à l'absence d'outils d'évaluation à l'époque de son élaboration en 1992. La disponiblité de certains de ces outils en 1999 (méthode des TAC èt indices d'abondance en jeunes saumons, stations de comptage des saumons adultes sur quelques bassins) rend possible un effort de quantification, bien que l'information biologique ne soit pas du même niveau de précision sur toutes les rivières.

A l'issue de sa mise en œuvre, on peut remarquer que les réalisations (en terme d'actions) sont proches du programme initial. La coordination multipartite du programme a permis de rétablir une approche plus synthétique. Parmi les principaux écarts à la prévision on peut citer la réalisation très incomplète d'ouvrages de franchissement, l'augmentation du poids des alevinages en saumon - en général transitoires - sur un petit nombre de bassins, et l'abandon de quelques actions d'accompagnement. L'organisation et le déroulement du programme se sont révélés satisfaisants pour l'ensemble des partenaires, ce qui a débouché sur l'intégration à part entière de la coordination en tant qu'élément central de la programmation.

Si l'on s'attache aux résultats biologiques concrets, on peut les qualifier:

- d'imperceptibles dans les cas où les actions entreprises sont incomplètes et ne pouvaient matériellement pas avoir eu d'effet à la fin de l'année 1999 (cas du saumon sur l'Aulne ou sur le Blavet);

- de mal ou pas connus dans un bon nombre de cas (Léguer, Trieux, etc.), ce qui plaide pour la poursuite de l'évaluation biologique ;

- de réels dans le cas de l'anguille sur la Vilaine ou le Frémur, ou encore du saumon sur l'axe Couesnon-Loysance, ce dernier projet ayant cependant débuté dès les années 1990.

L'évaluation des actions de communication est plus délicate, bien que le niveau de satisfaction des partenaires et du public à la plaquette "bilan " éditée en 1999 ainsi que la demande perceptible du public pour les sites de visualisation des migrations (Arzal, PontScorff et Châteaulin) soient susceptibles d'être appréciés dans un assez court délai.

Ces développements conduisent à poursuivre un bon nombre d'actions antérieures sur le programme 2000-2006, avec une certaine rationalisation issue du retour d'expérience du programme 1994-1999, tout en conservant la structure de partenariat mise en place en matière de coordination. Aussi, Ouest Grands Migrateurs, Groupement de l'Union Régionale des Fédérations et le Conseil Supérieur de la Pêche ont proposé la prise en compte d'autres espèces indicatrices - ou "repères " - de la qualité des milieux aquatiques, la truite commune et le brochet, permettant à terme la mise en ceuvre des préconisations formulées dans les Plans Départementaux de Protection des milieux aquatiques et de Gestion des ressources piscicoles (PDPG).

La principale fragilité du programme qui vient de s'achever est son caractère trop spécifique, son champ d'intervention assez restreint et sa vocation curative, d'où découle un risque de voir des améliorations contrecarrées par les modifications effectuées par 
ailleurs. Les aménagements agricoles des terres situées en tête de bassin ont pour effet de raréfier, d'altérer et de réchauffer l'eau des ruisseaux qui s'y trouvent, au détriment des populations de truites et indirectement sur celles de saumons. Il en va de même de la création de plans d'eaux sur les ruisseaux salmonicoles, dont l'impact est aussi négatif que sous-estimé par l'opinion publique, ou encore la réfection de sentiers s'accompagnant de busages pouvant se révéler être des obstacles insurmontables pour les truites sur ces mêmes ruisseaux. Des modifications profondes résultent fréquemment du cumul de petits aménagements insidieux, et les actions classiques des programmes «migrateurs " ne peuvent pas les traiter ni même les compenser véritablement. Si l'objectif global des programmes " migrateurs " est d'éviter les dégradations supplémentaires des milieux aquatiques courants, ils doivent atteindre -voire s'appuyer sur- les partenaires locaux capables d'influencer les décisions locales d'aménagement du territoire. Cet aspect préventif est l'une des interrogations du programme 2000-2006, qui souhaite non seulement s'ouvrir à des espèces piscicoles indicatrices nouvelles (truite et brochet), mais aussi avoir un effet en amont de certaines décisions à impact négatif.

\section{BIBLIOGRAPHIE}

ACOU A., 1999. Languille européenne : Dynamique et déterminisme de la migration catadrome dans un bassin versant anthropisé de Bretagne (Frémur). Université de Rennes I, $172 \mathrm{p}$.

ARAGO M.A., 2000. Bilan des actions «Poissons migrateurs " du contrat de plan EtatRégion 1994-1999 et propositions pour un programme « Rivières et poissons migrateurs " dans le contrat de plan Etat-Région 2000-2006. Ouest Grands Migrateurs Bretagne, 106 p. + annexes.

BRULARD J., 1995. Analyse économique de la pêche de loisir du saumon en Finistère. Fédération pour la Pêche et la Protection du Milieu aquatique du Finistère / Conseil Supérieur de la Pêche, Délégation Régionale de Bretagne-Basse-Normandie, $44 \mathrm{p}$.

BRULARD J., 1998. La pêche du saumon à la ligne sur l'Aulne en 1997. Suivi de l'effort de pêche et analyse économique de la pêche du saumon. Fédération pour la Pêche et la Protection du Milieu aquatique du Finistère, 80 p. + annexes.

PORCHER J.P., 1992. Poissons migrateurs - contrat « Retour aux sources ", propositions d'action- Ministère de l'environnement/Conseil Supérieur de la Pêche, 169 p.

PREVOST E., BAGLINIERE J.L., 1993. Présentation et premier éléments de mise au point d'une méthode simple d'évaluation du recrutement en juvéniles de saumon atlantique (Salmo salar) de l'année en eau courante, INRA, $10 \mathrm{p}$.

PREVOST E., PORCHER J.P., 1996. Méthodologie d'élaboration de Totaux Autorisés de Captures (TAC) pour le Saumon atlantique (Salmo salar L.) dans le Massif Armoricain. INRA/CSP, 15 p. + figures.

PREVOST E., PORCHER J.P., 1999. Note sur la situation et l'évolution récente du stock de saumons sur le bassin de l'Aulne (Finistère). Document de travail provisoire. $3 \mathrm{p}$. 